\title{
Viscous heating in fluids with temperature-dependent viscosity: implications for magma flows
}

\author{
A. Costa ${ }^{1,2}$ and G. Macedonio ${ }^{3}$ \\ ${ }^{1}$ Dipartimento di Scienze della Terra e Geologico-Ambientali, Università di Bologna, Italy \\ ${ }^{2}$ Dipartimento di Scienze della Terra, Università degli Studi di Pisa, Italy \\ ${ }^{3}$ Osservatorio Vesuviano, Istituto Nazionale di Geofisica e Vulcanologia, Napoli, Italy
}

Received: 5 March 2003 - Revised: 27 May 2003 - Accepted: 2 June 2003

\begin{abstract}
Viscous heating plays an important role in the dynamics of fluids with strongly temperature-dependent viscosity because of the coupling between the energy and momentum equations. The heat generated by viscous friction produces a local temperature increase near the tube walls with a consequent decrease of the viscosity which may dramatically change the temperature and velocity profiles. These processes are mainly controlled by the Peclét number, the Nahme number, the flow rate and the thermal boundary conditions. The problem of viscous heating in fluids was investigated in the past for its practical interest in the polymer industry, and was invoked to explain some rheological behaviours of silicate melts, but was not completely applied to study magma flows. In this paper we focus on the thermal and mechanical effects caused by viscous heating in tubes of finite lengths. We find that in magma flows at high Nahme number and typical flow rates, viscous heating is responsible for the evolution from Poiseuille flow, with a uniform temperature distribution at the inlet, to a plug flow with a hotter layer near the walls. When the temperature gradients induced by viscous heating are very pronounced, local instabilities may occur and the triggering of secondary flows is possible. For completeness, this paper also describes magma flow in infinitely long tubes both at steady state and in transient phase.
\end{abstract}

\section{Introduction}

A common feature of lava flows is the presence of a complex network of lava tubes, observed both in "pahoehoe" (Peterson et al., 1994) and 'a'a lava fields (Calvari and Pinkerton, 1998, 1999). The tube system acts as an efficient pathway for lava from the main vent to the flow fronts, allowing great distances to be reached. This is mostly due to the decrease of radiative cooling, as a consequence of a much lower temperature of the outer crust of the tubes as compared to the temper-

Correspondence to: A. Costa (costa@ov.ingv.it) ature of the lava surface flowing in open channels (Dragoni, 1989; Dragoni et al., 1995).

The effects of heat generation by viscous friction for lava flows in tubes and channels were often neglected in previous models. In this work we show that these effects can play an important role in the dynamics of fluids with strongly temperature-dependent viscosity such as silicate melts and polymers. In fact, in these fluids, viscous friction generates a local increase in temperature near the tube walls with consequent viscosity decrease and increase of the flow velocity. This velocity increase produces a further growth of the local temperature. As we will see later, there are some critical values of the parameters that control this process above which this feedback cannot converge. In this case the onedimensional laminar solution, valid in the limit of infinitely long pipe, cannot exist even for low Reynolds numbers. In pipes with finite length, viscous heating governs the evolution from Poiseuille regime with a uniform temperature distribution at the conduit inlet, to a plug flow with a hotter boundary layer near the walls downstream. This effect could be observed even in fluids with null yield strength. When the temperature gradients, induced by viscous heating are very pronounced, local instabilities occur and triggering of secondary flows is possible.

Viscous heating was previously invoked to describe the rheological behaviour of basalts (Shaw, 1969) and to explain some instabilities in the mantle (Anderson and Perkins, 1974; Zhao and Yuen, 1987; Larsen et al., 1995; Hansen and Yuen, 1996).

Moreover Fujii and Uyeda (1974), adopting a model only valid for infinitely long tubes (by the thermal point of view), explained the size of intrusive dikes in volcanoes.

Because of the low thermal conductivity of silicate melts, the temperature field shows a strong radial gradient and viscosity layering. Flows with layers of different viscosity were investigated in the past, also for their practical interest, and it is known that they are not always stable (Yih, 1967; Craik, 1969; Renardy and Joseph, 1985; Renardy, 1987). The instabilities are generated by the viscosity contrasts, and are sim- 
ilar to the Kelvin-Helmholtz instabilities triggered by density gradients. In Couette flows of fluids with temperaturedependent viscosity, Sukanek and Laurence (1974) and Yueh and Weng (1996) found one instability mode related to the viscosity gradient at low Reynolds numbers. This kind of instability shows a local character (Pearson, 1976) and is expected to occur also in lava flows.

As we will see later, these processes are controlled principally by three parameters: the Peclét number $(P e)$, the Nahme number ( $N a_{0}$, also called Brinkman number), and the non-dimensional flow rate $(q)$ :

$$
P e=\rho c U H / k ; \quad N a_{0}=\mu_{0} U^{2} \beta / k ; \quad q=\mu_{0} Q / \rho g H^{3}
$$

with $\rho$ density, $c$ specific heat, $U$ mean velocity, $H$ tube radius, $k$ thermal conductivity, $\mu_{0}$ reference viscosity $\left(N a_{0}\right.$ is based on this value), $\beta$ rheological parameter (see Eq. 5) and $Q$ flow rate per unit length $(Q=U H)$.

Viscous heating in lava and magma flows is responsible for effects not described by simple isothermal models, and may help in the understanding of some phenomena observed in lava flows, which are not yet clearly described. These include the surprising temperature increase at the base of pahoehoe flows observed by (Keszthelyi, 1995), the formation of the "roller vortex" (Booth and Self, 1973), thermal erosion at low Reynolds numbers (Greeley et al., 1998), and the observation, in lava channels, of magma temperatures greater than the eruption temperature at the vent (Kauahikaua et al., 1998). Erosion by viscous heating in mantle convection was also investigated by Larsen et al. (1997) and Moore et al. (1998).

Recently, the results of the studies of Polacci et al. (2001) suggest that the viscous heating may be responsible for the generation of a heterogeneous distribution of magma properties inside the conduit. Moreover, the recent viscous gravity currents model of Vasilyev et al. (2001), based on the conservation equations applied to a control volume, shows that the viscous dissipation exerts a strong influence in the viscous gravity currents. Finally, inadequacy of simple conductive cooling models is shown by basal temperature measurements carried out by Keszthelyi (1995). Temperature measurements recorded at the base of many flows increases after some initial cooling. This fact can be easily understood on the light of the model presented here.

We begin our study by investigating the limit case of flows in infinitely long tubes, and then we will describe the flow in tubes of finite length as being more representative of actual magma flows. We find that viscous heating effects cannot be generally neglected in typical magma flows and that the energy and momentum equations cannot be decoupled. Moreover, we find that the characteristic plug-like velocity profile observed in magma flows can be caused by viscous heating effects, even assuming Newtonian rheology.

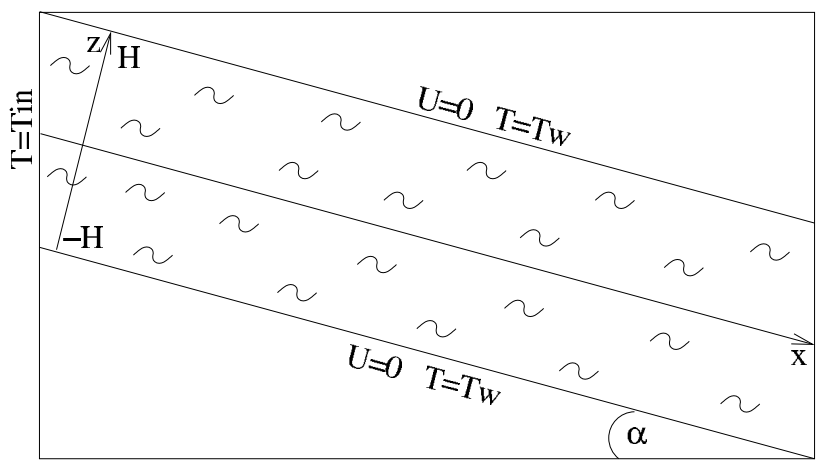

Fig. 1. Sketch of the inclined lava tube and prescribed boundary conditions.

\section{Model description}

In this paper, magma is assumed to be incompressible and approximated as an homogeneous fluid with constant density, specific heat and thermal conductivity. The viscosity, however, is temperature-dependent. We investigate the flow in a slab between two parallel boundaries separated by a distance $2 H$ and inclined of an angle $\alpha$ with respect to the horizontal (see Fig. 1). For symmetry, the velocity is directed along the flow. At the tube walls $(z= \pm H)$ we impose a null velocity and a fixed temperature. This last assumption on the temperature is quite restrictive, and will be probably generalized in future works. However, for the case of tube of finite length, we show also results obtained by assuming thermal adiabatic condition at the walls, as a limit case.

When the Peclét number is very high, the lubrication approximation is valid: the transient term in the momentum equation is orders of magnitude smaller than the corresponding term in the energy equation (their ratio is of the order of the inverse of the Prandtl number). This means that the time scale for momentum relaxation is much shorter that the corresponding time scale for thermal relaxation, and the time evolution of the velocity and temperature profiles is controlled only by the energy equation. In these hypotheses, magma dynamics in the tube are described by the following transport equations:

$$
-\frac{\partial P}{\partial x}+\rho g \sin \alpha+\frac{\partial}{\partial z}\left(\mu \frac{\partial U}{\partial z}\right)=0
$$

and

$\rho c\left(\frac{\partial T}{\partial t}+U \frac{\partial T}{\partial x}+W \frac{\partial T}{\partial z}\right)=k \frac{\partial^{2} T}{\partial z^{2}}+\mu\left(\frac{\partial U}{\partial z}\right)^{2}$

where $z$ is the coordinate transversal to the flow, $x$ longitudinal coordinate, $t$ time, $(U, W)$ velocity of the fluid in the directions $x$ and $z$ respectively, $\rho$ density, $P$ pressure, $\mu$ viscosity, $g$ gravity acceleration, $\alpha$ slope, $c$ specific heat at constant pressure, $T$ temperature, and $k$ thermal conductivity. The last term in Eq. (3) represents the heat generation by viscous dissipation. 
The characteristic length scales of this problem are the channel dimensions $2 H$ (thickness) and $L$ (length), the mechanical relaxation length $L_{m}=U H^{2} \rho / \mu_{0}$, and the thermal relaxation length $L_{t}=U H^{2} \rho c / k$. For lava flows, typically $L_{m} / L \ll 1$, but $L_{t} / L \gg 1$ and the approximation of infinitely long tube is not valid. Only when $L_{t} / L \ll 1$, the approximation of infinitely long tube (by the thermal point of view) is allowed. This limit case is simpler to handle, and it was widely studied in the past. For these reasons, we discuss magma flow in infinitely long tubes, before studying the viscous heating effects in tubes of finite length.

In silicate melts the dependence of the viscosity on the temperature is well described by the Arrhenius law (Shaw, 1969; Danes, 1972; Park and Iversen, 1984; Baloga and Pieri, 1986; Crisp and Baloga, 1994):

$\mu=\mu_{A} \exp (B / T)$

where $\mu_{A}$ is a constant and $B$ is the activation energy. In the interval of temperatures $\left(T-T_{0}\right) / T_{0} \ll 1\left(T_{0}\right.$ is a reference temperature), Eq. (4) is well approximated by the (Nahme's) exponential law:

$\mu=\mu_{0} \exp \left[-\beta\left(T-T_{0}\right)\right]$

with $\beta=B / T_{0}^{2}$ and $\mu_{0}=\mu_{A} \exp \left(B / T_{0}\right)$. In the following, for simplicity, we adopt the exponential law, more suited for the analytical manipulation of the equations (Shaw, 1969; Dragoni, 1989; Costa and Macedonio, 2002).

\subsection{Infinitely long pipe}

In this section we study the flow in an infinitely long channel (slab) driven only by the component of the gravity force acting along the flow (all gradients are null along the flow). We assume no-slip conditions $(U=0)$, constant temperature $\left(T=T_{w}\right)$ at the walls $(z= \pm H)$ and, for simplicity, we choose the reference temperature $T_{0}=T_{w}$. Under these hypotheses, Eqs. (2) and (3) reduce to:

$\rho g \sin \alpha+\frac{d}{d z}\left[\mu(T) \frac{d U}{d z}\right]=0$

and

$\rho c \frac{\partial T}{\partial t}=k \frac{\partial^{2} T}{\partial z^{2}}+\mu\left(\frac{d U}{d z}\right)^{2}$

In a previous work, Pearson (1977) considered a Poiseuille flow between two horizontal parallel planes with isothermal boundaries (the temperature at the boundaries is the same as that of the injected fluid), negligible transversal gradients, and a given flow rate. Previously, a similar problem (the Hagen-Poiseuille flow) was numerically solved using analogue computers by Gruntfest et al. (1964), and later Eckert and Faghri (1986) studied the Couette flow. Like Pearson (1977), Gruntfest et al. (1964) found that during the transient phase, in contrast to the steady-state, the temperature profile shows higher values near the walls than in the channel centre. Moreover, Gruntfest et al. (1964) found that the solutions of the equations that they consider do not diverge only when the non-dimensional pressure gradient $\mathcal{G}_{p}$ $\left(\mathcal{G}_{p}=\beta(d P / d x)^{2} H^{4} / k \mu_{0}\right)$ is smaller than a critical value. In fact for $\mathcal{G}_{p}$ greater than this value, the temperature increases indefinitely in a finite time. Gruntfest et al. (1964) suggest that the behaviour of this model can help in understanding the origin of turbulence (however this feedback could not produce an indefinite temperature increase in 2-D and 3-D systems).

Next we investigate the time-dependent problem of a fluid with initial temperature $T_{i}$, and wall temperature $T_{w}=T_{0}$, with $T_{i} \geq T_{0}$. We study the conditions for the existence of the solution, and the evolution to the steady-state solution (when it exists). After combining Eqs. (6) and (7), we rewrite the energy equation in the non-dimensional form:

$\begin{cases}\frac{\partial \theta}{\partial \tau}=\frac{\partial^{2} \theta}{\partial \zeta^{2}}+\mathcal{G} \zeta^{2} e^{\theta} & \\ \theta=0 & \forall \tau>0, \zeta= \pm 1 \\ \theta=\mathcal{B} & \tau=0, \quad-1<\zeta<1\end{cases}$

where

$\theta=\beta\left(T-T_{0}\right) ; \quad \zeta=\frac{z}{H} ; \quad \tau=k t /\left(\rho c H^{2}\right)$

$\mathcal{G}=\frac{\beta(\rho g \sin \alpha)^{2} H^{4}}{k \mu_{0}} ; \quad \mathcal{B}=\beta\left(T_{i}-T_{0}\right)$.

The parameter $\mathcal{G}$ represents the non-dimensional "shear stress", and is known as the Nahme number based on the characteristic stress (instead of the velocity).

From the results of Gruntfest et al. (1964) we know that when $\mathcal{B}=0$ and $\mathcal{G}>\mathcal{G}_{\text {crit }}\left(\mathcal{G}_{\text {crit }} \approx 5.64\right.$ for slab flows), the solution of Eq. (8) diverges in a finite time. In the case of $\mathcal{G}<\mathcal{G}_{\text {crit }}$, the solution of the transient phase, with two maxima near the walls, evolves for $\tau \approx 1$ to the steady-state solution with only one maximum in the channel centre. In Fig. 3 and Fig. 2 we report the temporal evolution of $\theta$ found numerically for the subcritical condition $(\mathcal{G}=5)$, and for the supercritical condition $(\mathcal{G}=8)$ respectively, both with $\mathcal{B}=0$. As shown in Fig. 3, when $\mathcal{G}<\mathcal{G}_{\text {crit }}$, for small $\tau$ the temperature increases near the boundaries and for $\tau \approx 1$ its profile tends to the steady-state solution. In Fig. 2, in the supercritical case, the temperature profile, shows two peaks near the walls that continuously increase, until they diverge when $\tau$ reaches a critical value $\tau_{\text {crit }}$.

The general case, with the initial fluid temperature greater than the temperature at the walls $(\mathcal{B}>0)$, was previously studied by Fujita (1969) from a theoretical point of view, but is not yet analytically or numerically solved (to our knowledge). Fujita (1969) clarified the relationship between the transient and the steady-state phases with some theorems for the steady-state boundary value problem:

$\nabla^{2} u+e^{u}=0 \quad(x \in \Omega)$

and for the corresponding time-dependent problem:

$\frac{\partial u}{\partial t}=\nabla^{2} u+e^{u} \quad(t \geq 0, x \in \Omega)$ 


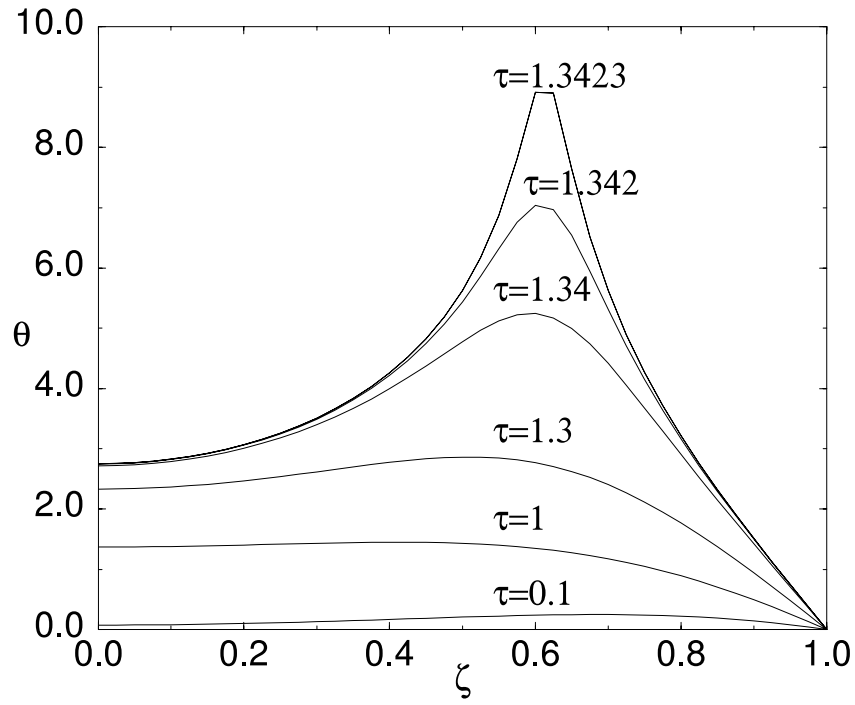

Fig. 2. Temporal evolution of the non-dimensional temperature profile in the supercritical condition $\mathcal{G}=8$ and $\mathcal{B}=0$.

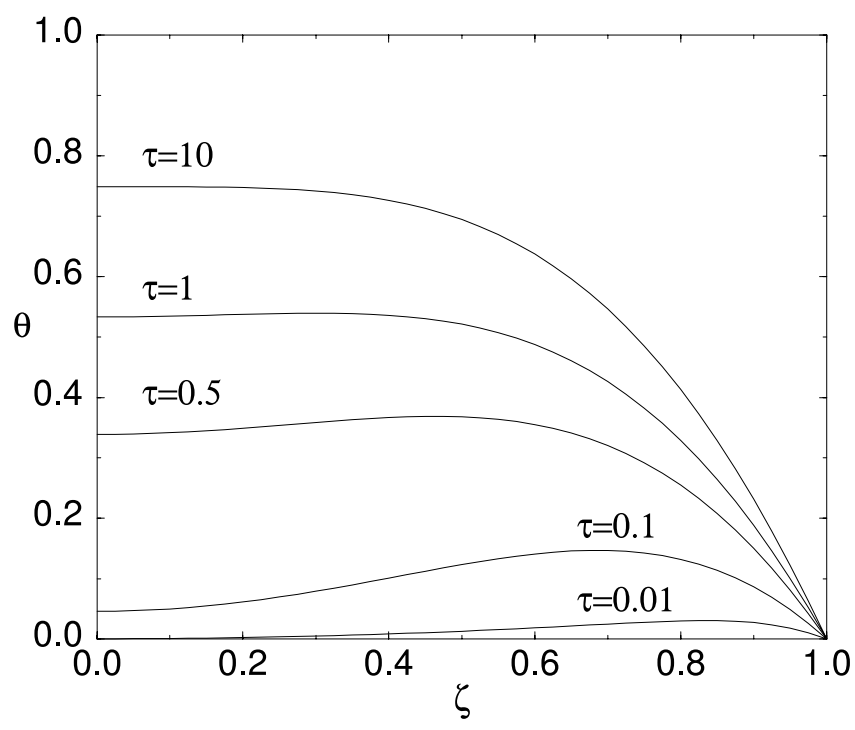

Fig. 3. Temporal evolution of the non-dimensional temperature profile in the subcritical condition $\mathcal{G}=5$ and $\mathcal{B}=0$.

with initial condition $u(t=0)=a(x)$ (with $a(x)$ continuous), and boundary condition $u(\partial \Omega)=0$. One of the theorems states that if Eq. (11) has no solution, then the solutions of Eq. (12) diverge in a finite time, or diverge for $t \rightarrow \infty$. Another theorem states that if Eq. (11) has more than one solution, $\phi$ is the "smaller" solution, and $\psi$ is another solution not equal to $\phi$, then:

1. If $a>\psi$, then the solution $u$ of Eq. (12) diverges, in a finite time, or for $t \rightarrow \infty$.

2. If $a<\psi$, then the solution $u$ of Eq. (12) uniformly converges to $\phi$, for $t \rightarrow \infty$.

From our numerical solutions of Eq. (8), we observe that when $\mathcal{B}>0$, the range of values of $\mathcal{G}$ below which the so-

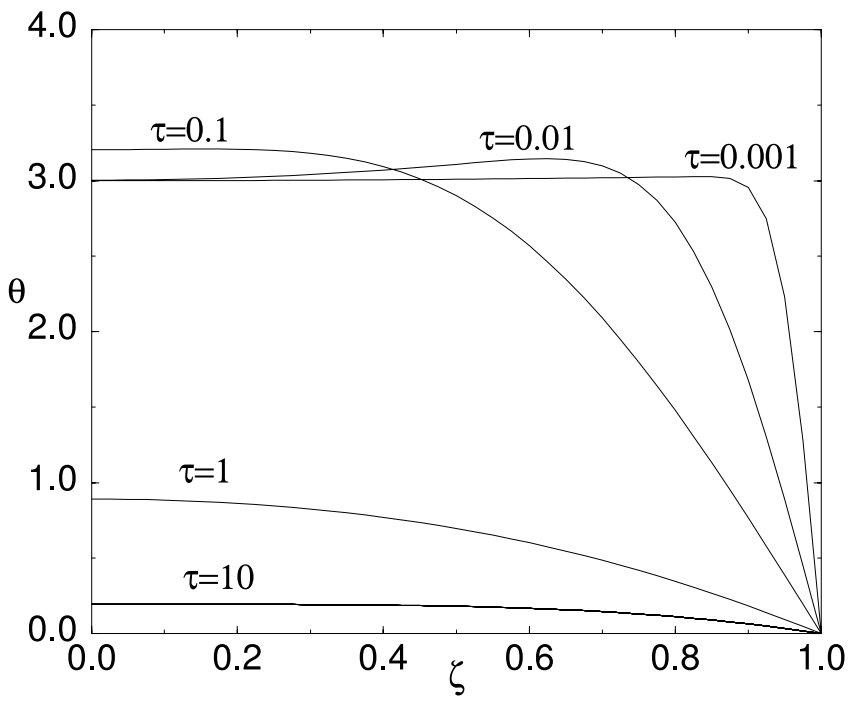

Fig. 4. Temporal evolution of the non-dimensional temperature for $\mathcal{G}=2$ and $\mathcal{B}=3$.

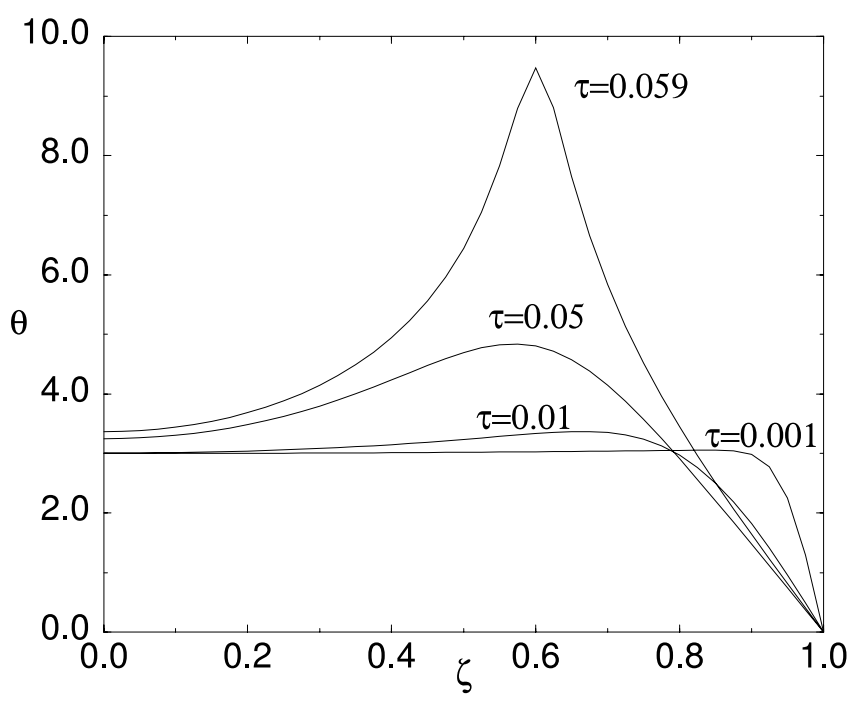

Fig. 5. Temporal evolution of the non-dimensional temperature profile in the supercritical condition $\mathcal{G}=4$ and $\mathcal{B}=3$.

lution of Eq. (8) converges is smaller than the corresponding range of $\mathcal{G}$ for $\mathcal{B}=0$, but the profiles evolution is similar to the case with $\mathcal{B}=0$. We call $\mathcal{G}_{\text {crit }}(\mathcal{B})$ the critical value of $\mathcal{G}$ above which the solution diverges when the initial condition is $\mathcal{B}$. An example is reported in Fig. 5 for $\mathcal{G}=4$ and $\mathcal{B}=3$ where the temperature diverges in a finite time. Figure 6 shows the relation between $\mathcal{B}$ and $\mathcal{G}_{\text {crit }}(\mathcal{B})$ found numerically. For $\mathcal{B}>0$ we study the following two cases: in the subcritical case $\left(\mathcal{G}<\mathcal{G}_{\text {crit }}\right)$ we investigate the effect of the viscous heating on the temporal evolution of the temperature profile, and we compare the results to the case without heat generation $(\mathcal{G}=0)$; in the supercritical case, we investigate the relation between the critical time corresponding to the thermal blow-up $\tau_{\text {crit }}$ and $\mathcal{G}$. When the viscous heating is neglected, Eq. (8) reduces to the classical heat equation. In fact 


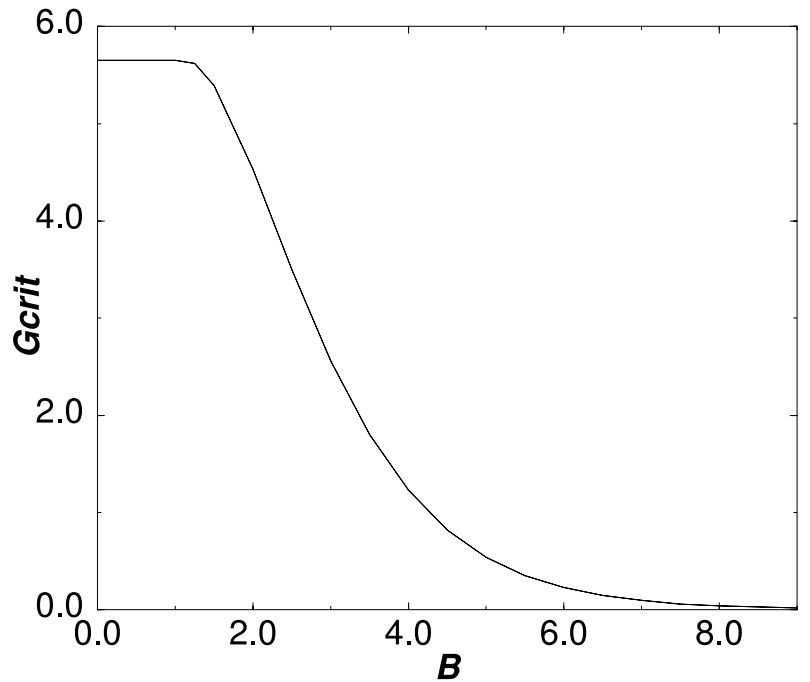

Fig. 6. Relation between $\mathcal{B}$ and $\mathcal{G}_{\text {crit }}(\mathcal{B})$.

from an initial condition with uniform temperature greater than that of the boundaries, the fluid starts cooling near the walls, until the wall temperature is reached in a time scale of the order of the characteristic time, i.e $\tau \sim 1$. By accounting for the viscous heating, as shown, for example, in Fig. 4, the temporal evolution of the temperature can be described in four phases. The initial phase is characterized by the cooling near the walls; in the second phase the viscous heating near the walls produces the increase of the temperature above its initial value and the temperature profile assumes two maxima in the external part of the profile and a minimum in the central part. During the third phase the two maxima migrate towards the central part of the channel, and the profile is characterized by a maximum temperature in the centre, that could be also greater than its initial value. In the fourth phase the fluid cools until the steady state solution is reached, in agreement with the theoretical results of Fujita (1969), previously reported.

In the supercritical case $\left(\mathcal{G}>\mathcal{G}_{\text {crit }}\right)$, we find an nondimensional critical time $\tau_{\text {crit }}$ above which the solution diverges. In this case, the temporal evolution of the temperature profile is qualitatively similar to the corresponding case with $\mathcal{B}=0$, but with shorter $\tau_{\text {crit }}$. The temperature increases near the walls until it diverges as $\tau \rightarrow \tau_{\text {crit }}$ (similar to the case shown in Fig. 5). As $\mathcal{G}$ increases, $\tau_{\text {crit }}$ gets shorter. As an example, Fig. 7 shows the relation between $\mathcal{G}$ and $\tau_{\text {crit }}$, for $\mathcal{B}=0$.

From the physical point of view, when viscous heating is relevant, a transversal temperature gradient develops near the walls to dissipate the heat through the boundaries. This temperature increase produces a viscosity decrease with a consequent increase of the flow velocity. This velocity increase produces an increase in the velocity gradients near the walls, and a further local temperature increase. When $\mathcal{G}>\mathcal{G}_{\text {crit }}$, the temperature increases without bound this feedback cannot converge and the steady-state solution does not exist. Of course, this has no physical meaning, and is related to the

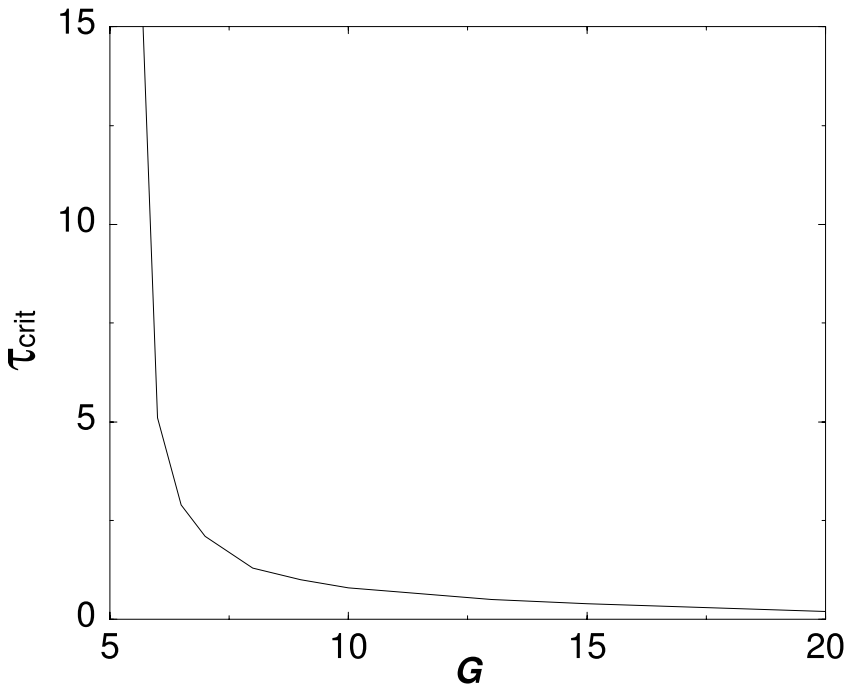

Fig. 7. Relation between $\tau_{\text {crit }}$ and $\mathcal{G}$ for $\mathcal{B}=0$.

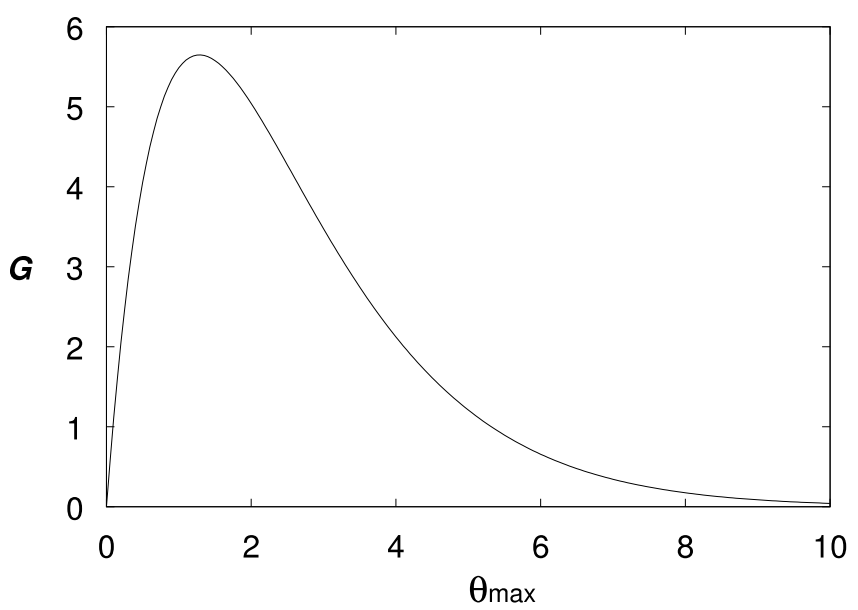

Fig. 8. Non-Dimensional "shear-stress" $\mathcal{G}$ vs. $\theta_{\max } .\left(\theta_{\max }\right.$ is the maximum temperature in the channel centre, at steady state.)

loss of validity of some assumptions such as the hypothesis of one-dimensional laminar flow. When the infinitely long pipe hypothesis is valid, according to Gruntfest et al. (1964), it is legitimate to assume that above the critical values $\left(\mathcal{G}_{\text {crit }}\right.$, $\tau_{\text {crit }}$ ), other diffusive processes are triggered, allowing a more efficient heat and momentum transfer.

Instead, when $\mathcal{G}<\mathcal{G}_{\text {crit }}$, the temperature and velocity profiles tend to the steady-state solutions described by:

$\frac{d^{2} \theta}{d \zeta^{2}}+\mathcal{G} e^{\theta} \zeta^{2}=0$

Eq. (13) was widely studied in the past, and it is well known that it has no solutions for $\mathcal{G}>\mathcal{G}_{\text {crit }}$, where $\mathcal{G}_{\text {crit }}$ is a critical value (Joseph, 1964). Below the critical value, for each $\mathcal{G}$ there are two solutions corresponding to different temperature profiles: one at low temperature, and one at high temperature (see Fig. 8). For some years, the existence of multiple solutions corresponding to a given "shear-stress" $\mathcal{G}$ was discussed in the literature, and the stability of the solu- 
tion in the higher branch was investigated. This depends on which is the controlling variable of the problem: the velocity or the shear stress (John and Narayanan, 1997). Moreover, the relevance of the boundary conditions to the multiple steady-states and the stability of the solution was investigated in the contest of asthenospheric shear flow (Yuen and Schubert, 1977). A complete review on this argument until 1974 is reported by Sukanek and Laurence (1974), and more recently by Becker and McKinley (2000). In summary, in the Poiseuille flow between two parallel planes, when the non-dimensional "shear-stress" parameter $\mathcal{G}$ is greater than a critical value $\mathcal{G}_{\text {crit }}(\simeq$ 5.64), Eq. (13) has no solutions; for $\mathcal{G}=\mathcal{G}_{\text {crit }}$ it has one solution; and for $\mathcal{G}<\mathcal{G}_{\text {crit }}$ it has two solutions, one of which (the one with greater temperature) may be unstable. For $\mathcal{G}<\mathcal{G}_{\text {crit }}$, typical profiles of the nondimensional temperature $\theta$, and velocity $u / U_{0}$ that satisfy Eq. (6), Eq. (7) and the imposed boundary conditions, are characterized by a maximum in the centre of the tube.

\subsection{Finite length tube}

From the thermal point of view, magma flows at high Peclét number cannot be described by assuming infinitely long tubes. At high Peclét numbers, the leading term in the left side of Eq. (3) is the advective term, containing the longitudinal gradient of the temperature (e.g. Lawal and Kalyon, 1997). Including only the relevant terms, the transport equations (mass, momentum and energy balance) for finite length tubes, in non-dimensional form are:

$$
\begin{aligned}
& \int_{0}^{1} u d \zeta=q \\
& \frac{\partial u}{\partial \zeta}=\left(\frac{\partial p}{\partial \xi}-\sin \alpha\right) \zeta e^{\theta} \\
& P e^{*} u \frac{\partial \theta}{\partial \xi}=\frac{\partial^{2} \theta}{\partial \zeta^{2}}+N a^{*}\left(\frac{\partial u}{\partial \zeta}\right)^{2} e^{-\theta} \text { (energy) }
\end{aligned}
$$

where $u=U / U^{*}$ with $U^{*}=\rho g H^{2} / \mu_{0}$, and $p$ nondimensional pressure $(p=P / \rho g H)$; moreover:

$q=\frac{\mu_{0} Q}{\rho g H^{3}}$

$P e^{*}=\frac{\rho^{2} c g H^{3}}{k \mu_{0}}=\frac{P e}{q}$

$N a^{*}=\frac{\beta \rho^{2} g^{2} H^{4}}{k \mu_{0}}=\frac{N a_{0}}{q^{2}}$

where $N a^{*}$ and $P e^{*}$ are the Nahme and the Peclét numbers based on the characteristic velocity $U^{*}$ respectively and $Q=U H$. The equation describing mass conservation is written in integral form. To solve the equations in (14), we assume no-slip conditions for the velocity and constant temperature $T_{w}$ at the walls. At the inlet we assume a parabolic velocity profile and constant temperature $T_{i n}$. Here, for simplicity and according to other authors, we consider the wall temperature as reference, i.e. $T_{0}=T_{w}$ and $\mathcal{B}=\beta\left(T_{i n}-T_{w}\right)$. From an operative point of view, it would probably be more convenient to set the reference temperature equal to the temperature at the inlet. Of course, the physical behaviour of the flow is independent of the choice of the reference temperature $T_{0}$; this is obtained by rescaling all the parameters. As an example, by assuming $T_{0}=T_{i n}$, the viscosity needs to be rescaled by a factor $e^{\mathcal{B}}$, etc.

Recently, Lawal and Kalyon (1997) found analytical solutions of the simpler problem with constant viscosity and showed typical temperature profiles with two peaks near the walls. In our case, since we consider temperature-dependent viscosity, we expect a feedback on the velocity profile. In fact, using asymptotic solution method for high Nahme numbers, Ockendon (1979) solved the Eq. (14) taking in to consideration the full advective term. These solutions describe the evolution of a flow from the Poiseuille regime with uniform temperature at the conduit inlet to a plug regime characterized by two peaks in the temperature profile near the boundaries far from the inlet. Since the ratio $L_{t} / L_{m}$ (equal to the Prandtl number) is very high, it is legitimate to assume that the flow is fully developed, by the mechanical point of view, almost at the tube inlet. The characteristic distance from the inlet where the viscous heating becomes relevant, is $\xi^{*} \sim \mathrm{PeNa}^{-3 / 2}$ (Ockendon, 1979) (using data from Table 1 this gives $50 \div 5000 \mathrm{~m}$ for a conduit width of $5 \mathrm{~m}$ ). Under more general conditions, Schneider (1976) found numerical solutions of the viscous heating problem, showing clearly that the deviation from the Newtonian behaviour may have a thermal origin in fluids with temperature-dependent viscosity. When the viscous dissipation becomes important, its effect overcomes the thermal cooling from the walls producing a maximum in the temperature profile near the walls and a consequent plug-like velocity distribution. These effects increase with distance from the inlet and, unexpectedly, the maximum temperature gets more pronounced when the temperatures at the walls get lower. Similar results were obtained by Galili et al. (1975) using perturbative methods: they found that the flow rate is not proportional to the longitudinal pressure gradient and the apparent viscosity of a Newtonian fluid depends on the shear rate because of the viscous dissipation.

We solve Eq. (14) by using a finite-difference method with an implicit scheme for the integration along direction $\xi$; the pressure gradient is iteratively adjusted at each step in order to satisfy mass conservation. For symmetry we investigate only half a channel $(0 \leq \zeta \leq 1)$. We find that the process is controlled by four parameters: a Peclét number $P e$, a Nahme number $N a^{*}$, the non-dimensional flow rate $q$, and the nondimensional input temperature $\mathcal{B}$. However, since we focus on magma flows in tubes, we set $P e=10^{7}$ as a typical value, and we perform a parametric study on the others.

In Fig. 9 and 10, we show a solution of Eq. (14) for different non-dimensional distance from the inlet: from $\xi=0$ to $\xi=10^{4}$. Starting with uniform temperature $(\theta=0)$ and parabolic velocity profile at the inlet, the flow evolves gradually to a plug-like velocity profile with two symmetric peaks in the temperature distribution. In this case we used 


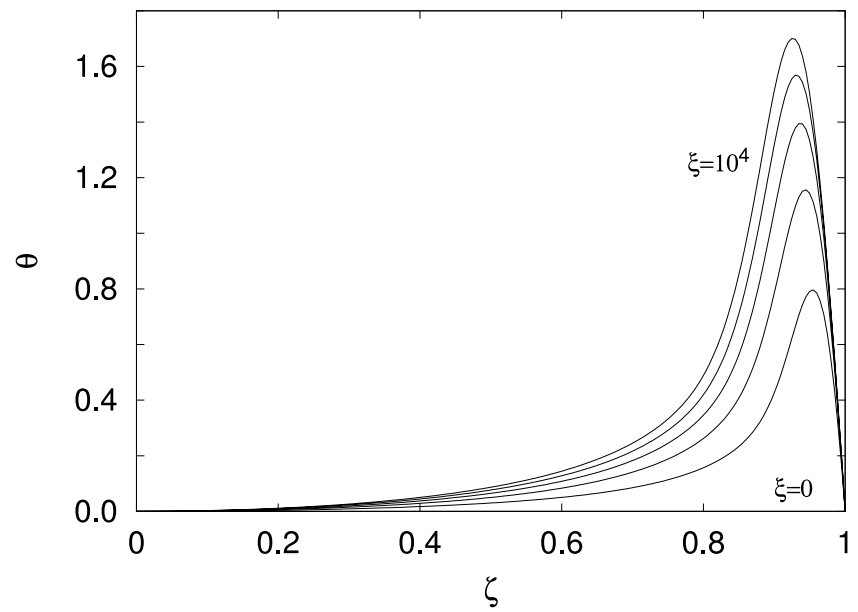

Fig. 9. Longitudinal evolution of the non-dimensional temperature profile $P e=10^{7}, N a^{*}=100, q=1, \mathcal{B}=0$ for different nondimensional distance from the inlet $\left(10^{-3} \xi=0,2,4,6,8,10\right)$.

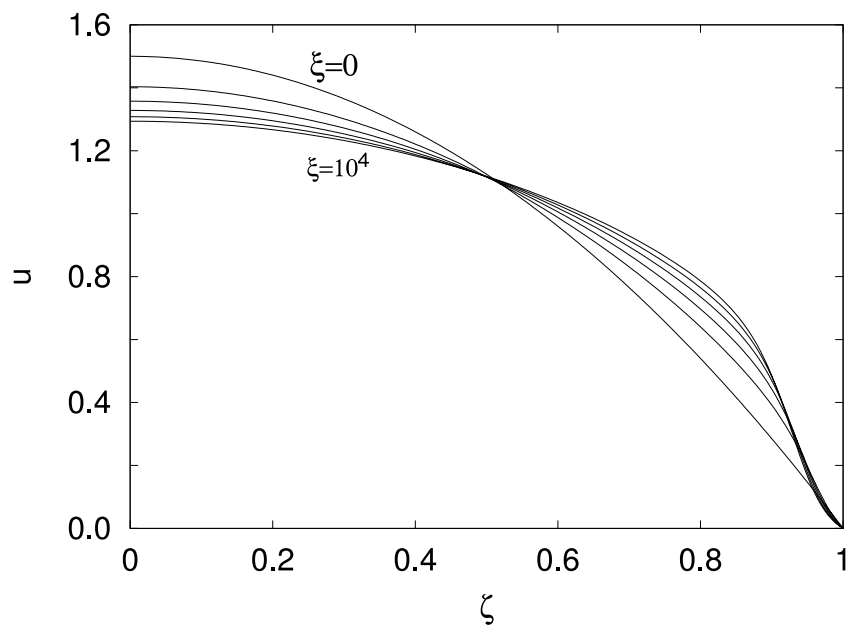

Fig. 10. Longitudinal evolution of the non-dimensional velocity profile $P e=10^{7}, \mathrm{Na}^{*}=100, q=1, \mathcal{B}=0$ for different nondimensional distance from the inlet $\left(10^{-3} \xi=0,2,4,6,8,10\right)$.

$N a^{*}=100, q=1$ and $\mathcal{B}=0$ and the viscous dissipation effects become important only for high values of $\xi$. Instead in Fig. 11 and 12 the solutions for $N a^{*}=1000, q=1$ and $\mathcal{B}=0$ are shown; these results are qualitatively similar to the previous ones but the temperature peaks are more pronounced and the length scale for the development of the plug flow is lower than before (practically, the transition occurs for $\xi=1000$ ).

When the viscous heating is important with $\mathcal{B}>0$, the typical temperature profile shows low values at the walls with peaks near the walls, as reported in Fig. 13. As shown in Fig. 14, starting with a parabolic velocity profile at the inlet $(\xi=0)$, the flow migrates to a plug-like regime downstream. By increasing $\mathcal{B}$, the peak in the temperature profile moves towards the centre of the channel.

In general, viscous heating becomes important when either the Nahme number $\left(\mathrm{Na}^{*}\right)$ or the non-dimensional flow

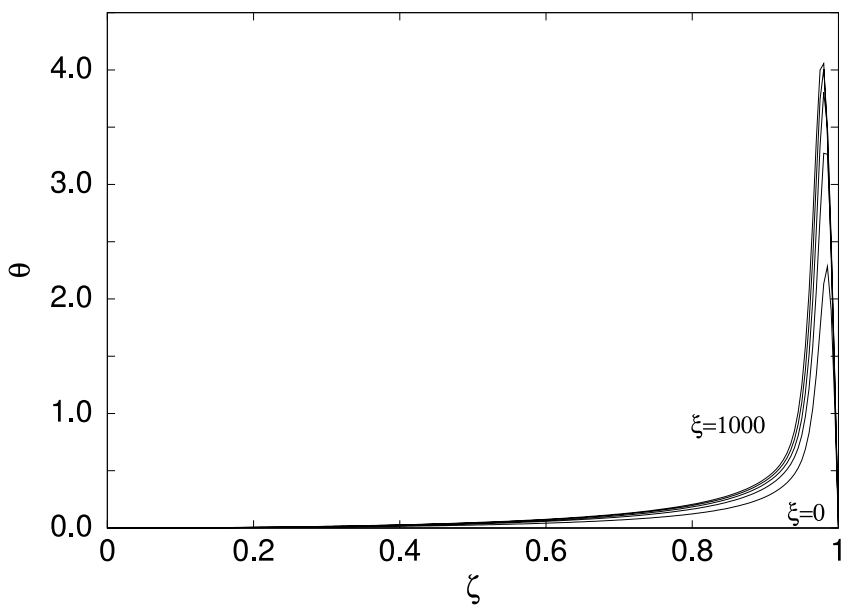

Fig. 11. Longitudinal evolution of the non-dimensional temperature profile $P e=10^{7}, N a^{*}=1000, q=1, \mathcal{B}=$ 0 for different non-dimensional distance from the inlet $(\xi=$ $0,200,400,600,800,1000)$.

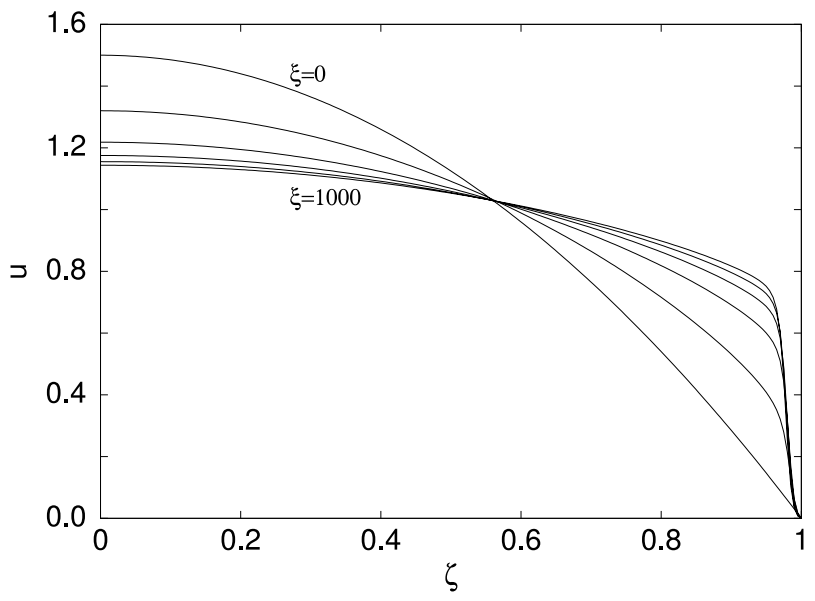

Fig. 12. Longitudinal evolution of the non-dimensional velocity profile $\mathrm{Pe}=10^{7}, N a^{*}=1000, q=1, \mathcal{B}=$ 0 for different non-dimensional distance from the inlet $(\xi=$ $0,200,400,600,800,1000)$.

rate $(q)$ increase. For comparison, in Fig. 15 and 16, we show the evolution of the temperature and velocity profiles in a case similar to Fig. 9 and 10, but with adiabatic thermal conditions at the walls. In this case, as expected, the temperature increases more than the case of isothermal walls, and the the velocity profile shows a more pronounced plug-like behaviour.

\subsection{Velocity field stability and secondary flows}

The energy equation of fluids with temperature-dependent viscosity in infinitely long flows is similar to the equation which governs chemical explosive processes in some materials (described by the Frank-Kamenetski equation, 1939). In this last case, when the parameter corresponding to $\mathcal{G}$ is greater than its critical value, ignition occurs. In fluids, however, other multidimensional degrees of freedom can be ac- 


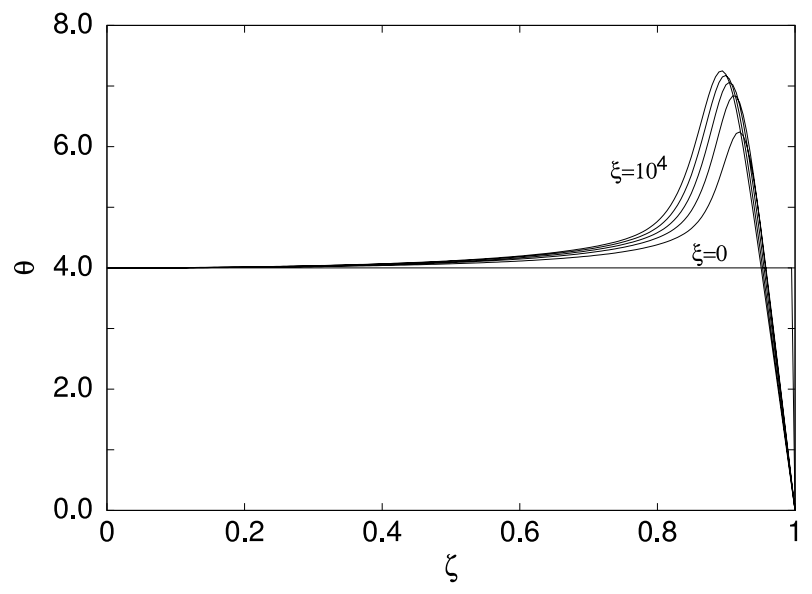

Fig. 13. Longitudinal evolution of the non-dimensional temperature profile $\mathrm{Pe}=10^{7}, \mathrm{Na}^{*}=1000, q=4, \mathcal{B}=4$ for different nondimensional distance from the inlet $\left(10^{-3} \xi=0,2,4,6,8,10\right)$.

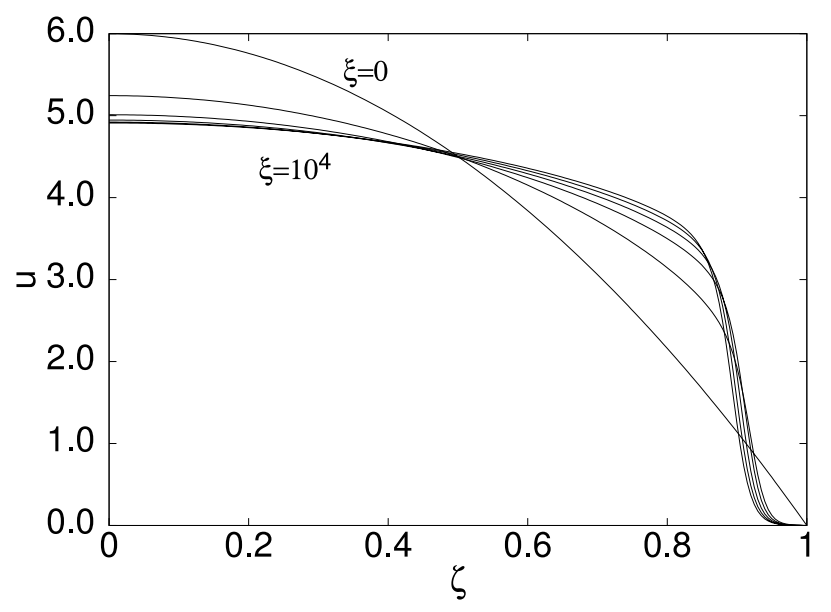

Fig. 14. Longitudinal evolution of the non-dimensional velocity profile $P e=10^{7}, N a^{*}=1000, q=4, \mathcal{B}=4$ for different nondimensional distance from the inlet $\left(10^{-3} \xi=0,2,4,6,8,10\right)$.

tivated without the occurrence of extreme events. For example, in flows between two parallel planes, cylindrical secondary flows can develop near the walls, or toroidal secondary flows can occur in circular pipes.

Due to the strong coupling between viscosity and temperature, the thermal instability generated by viscous heating may trigger an instability in the velocity field, which cannot be predicted by a simple isothermal newtonian models. When the viscous heating produces sharp peak in the temperature profile near the walls, with consequent strong increase in the viscosity gradient, a triggering of instabilities and the transition to secondary flows (with more efficient thermal and mechanical diffusion) is possible. The stability of the plane Couette flow was recently re-examined by Yueh and Weng (1996), who improve the results previously found by Sukanek et al. (1973). The flow shows two different instability modes: the former arises in the non-viscous limit, and the latter is due to the viscosity stratification (Yueh and

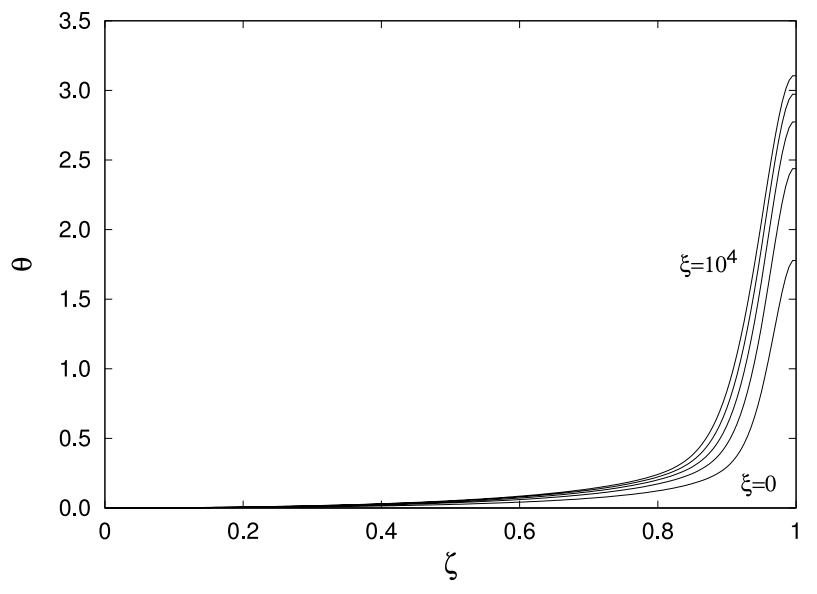

Fig. 15. Longitudinal evolution of the non-dimensional temperature profile $P e=10^{7}, \mathrm{Na}^{*}=100, q=1$, adiabatic walls, for different non-dimensional distance from the inlet $\left(10^{-3} \xi=0,2,4,6,8,10\right)$.

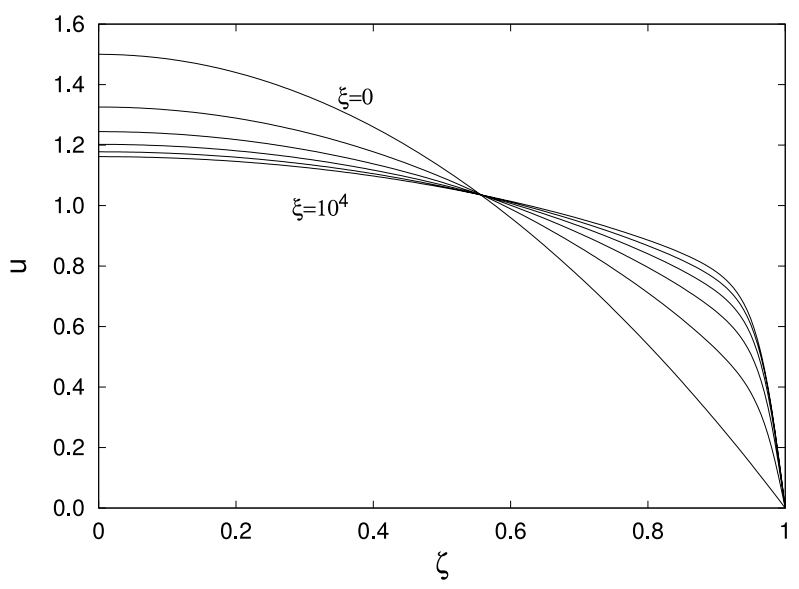

Fig. 16. Longitudinal evolution of the non-dimensional velocity profile $P e=10^{7}, \mathrm{Na}^{*}=100, q=1$, adiabatic walls, for different non-dimensional distance from the inlet $\left(10^{-3} \xi=0,2,4,6,8,10\right)$.

Weng, 1996). For this last instability mode, it was numerically demonstrated that the critical Reynolds number $\left(R e_{c}\right)$, above which the flow becomes turbulent, decreases with the increase of the Nahme number $(\mathrm{Na})$, that is with the viscous heating. This behaviour is confirmed by recent experiments performed by White and Muller (2000). In these experiments, the authors use a temperature-dependent fluid (glycerin) and a Taylor-Couette device which allows the tracking of vortex by a laser particle tracer. Results clearly show that, above a critical Nahme number, an instability appears at a Reynolds number one order of magnitude lower than the corresponding Reynolds number predicted for isothermal flow.

Moreover, the triggering of the instability in the laminar flow and the activation of new secondary flows are confirmed by our 2-D direct numerical simulations of the complete Navier-Stokes equations, which will be the subject of a further work. 
Table 1. Parameters characteristic of lava flows.

\begin{tabular}{ccc}
\hline$\rho$ & 2500 & $\mathrm{~kg} / \mathrm{m}^{3}$ \\
$\beta$ & 0.04 & $\mathrm{~K}^{-1}$ \\
$c$ & 1000 & $\mathrm{~J} \mathrm{~kg}^{-1} \mathrm{~K}^{-1}$ \\
$k$ & 2.0 & $\mathrm{~W} \mathrm{~m}^{-1} \mathrm{~K}^{-1}$ \\
$T_{S}$ & 1173 & $\mathrm{~K}$ \\
$T_{i n}$ & 1373 & $\mathrm{~K}$ \\
$\mu\left(T_{i n}\right)$ & 500 & $\mathrm{~Pa} \mathrm{~s}$ \\
$U$ & $1 \div 10$ & $\mathrm{~m} / \mathrm{s}$ \\
$2 H$ & $0.5 \div 5$ & $\mathrm{~m}$ \\
\hline
\end{tabular}

\section{Implications for lava flows}

In this section we apply the previous theoretical results to the study of lava and magma flows in tubes. We take the typical magma parameters reported in Table 1 as representative, but the obtained results can be easily generalized.

Following Bruce and Huppert (1989), $T_{w}$ is the temperature at the wall, which is defined as the temperature at which crystallization of magma ceases the flow. Here, we estimate $T_{w}$ as the temperature in the mid-range between $T_{i n}$ and the solidification temperature $T_{s}$ : therefore, we use $T_{0}=T_{w} \approx$ $1273 \mathrm{~K}$. From this value of $T_{w}$ and from the values of $\beta$ and $T_{i n}$, reported in Table 1 , we have $\mathcal{B}=\beta\left(T_{i n}-T_{w}\right) \approx 4$ and $\mu_{0}=\mu\left(T_{i n}\right) \cdot e^{\mathcal{B}} \approx 30,000$ Pas. Using the other values reported in Table 1, we obtain the following characteristic non-dimensional numbers:

$$
\begin{array}{ll}
q=\mu_{0} Q / \rho g H^{3} & 1 \div 10 \\
N a_{0}=\mu_{0} U^{2} \beta / k & 6 \cdot 10^{2} \div 6 \cdot 10^{4} \\
P e=\rho c U H / k & 3 \cdot 10^{5} \div 3 \cdot 10^{7} \\
P r_{0}=c \mu_{0} / k & 1.5 \cdot 10^{7} \\
N a=\mu\left(T_{i n}\right) U^{2} \beta / k & 1 \div 10^{3} \\
\operatorname{Re}=\rho U H / \mu\left(T_{i n}\right) & 1 \div 100 \\
\operatorname{Pr}=c \mu\left(T_{\text {in }}\right) / k & 3 \cdot 10^{5}
\end{array}
$$

The last three numbers in Eq. (18) are the Nahme, the Reynolds and the Prandtl numbers based on $\mu\left(T_{i n}\right)$, respectively, and are reported just for completeness.

The values of the non-dimensional numbers reported in Eq. (18) allow for the application of the model described above for lava and magma flows. In fact, the high Peclét number results in a very fast advective transport and validates the assumption of the lubrication theory. Concerning the effects of viscous heating on magma flows, we found that for the lower value of the non-dimensional flow rate considered in our study $(q=1)$ and $N a^{*}=N a_{0} / q^{2} \lesssim 1000$, the cooling effects prevail on the viscous heating. This becomes dominant for $\mathrm{Na}^{*} \sim 10^{4}$ showing velocity and temperature profiles similar to those reported in Fig. 13 and 14. Instead, at high flow rates $(q=10)$, the corresponding values of $\mathrm{Na}^{*}$ are lower: the viscous heating effects are dominant even at $N a^{*} \sim 100$ whereas for $N a^{*} \lesssim 10$ cooling effects prevail. Since these conditions are usual in magma flows, it should be possible to observe viscous heating effects in the natural environment.

For magma flows in eruptive conduits, recently, Polacci et al. (2001) emphasized the importance of the viscous heating in magma flow during volcanic eruptions to explain the creation and the discharge of two different varieties of pumice. In their scheme, one type of pumice originates in the region with greater temperature and higher strain rate near the conduit walls, whereas the other type is generated in the central part of the conduit with lower strain rate and temperature.

For lava flows, field evidences corresponding to the starting of secondary flows, previously described, are possibly represented by the "roller vortex" phenomenon (Booth and Self, 1973) and by the thermal erosion observed by Greeley et al. (1998) where the authors find "unequivocal evidence for thermal erosion" in lava tubes at Cave Basalt, Mt. St. Helens for which the dynamic analysis indicates laminar flows. It is known that the turbulent flow is more effective in erosion than the laminar flow because of the higher heat transfer rate (Hulme, 1973; Greeley et al., 1998); moreover preliminary studies show that purely thermal erosion by laminar flow is very difficult unless the substrate is of much lower melting temperature than the eroding fluid (Greeley et al., 1998). Field studies report that during the Kilauea eruption in 1994, lava eroded $5 \mathrm{~m}$ of the basaltic substrate with an average erosion rate of $10 \mathrm{~cm} /$ day at low Reynolds numbers between 16 and 64 (Kauahikaua et al., 1998). Such high erosion rates highlight the difficulties that arise when trying to explain thermal erosion in laminar flows. Moreover, Kauahikaua et al. (1998) show that the Jeffreys equation that relates the velocity and the flow depth in a laminar flow is less adequate than other relations valid for turbulent flows such as the equation of Goncharov-Chezy, even for flows at low Reynolds numbers. Concerning the temperature, Kauahikaua et al. (1998) report the interesting and puzzling observations of some temperature measurements greater than the magma temperature at the vent and even greater that the upper limit of the temperature range of the used radiometer (about $1200^{\circ} \mathrm{C}$ ). An explanation of this phenomenon could be the local increase of temperature near the walls caused by the viscous heating, as previously described. Other authors also report the evidence of vortices associated with the beginning of turbulence in active lava flows (Keszthely and Self, 1998) and the onset of complex flow patterns Greeley (1987); Lipman and Banks (1987); Crisp and Baloga (1994); Calvari and Pinkerton (1998). The inadequacy of pure laminar flows to explain field observation such as mixing and streamlines breaking has been well known since the sixties when the not widely accepted term of "disrupted flow" was introduced to describe a flow with characteristics between pure laminar and turbulent (for a discussion see Baloga et al., 1995). Moreover basal temperature measurements carried out by Keszthelyi (1995) show that temperature at the base of some flows increases after some initial cooling. This behaviour cannot be predicted by a simple conductive model but can be explained using a correct physical model which correctly describes vis- 
cous heating effects.

At Etna, during the eruption of 1971, secondary rotational flows were observed near the walls in flows confined between levees or in deep channels (Booth and Self, 1973). These secondary flows consist of two elicoidal patterns symmetrical to the centre of the flow. Booth and Self (1973), because of the low Reynolds number, exclude any turbulent flow, and try to explain these rotational flows invoking other causes and other, sometimes weak, argumentations. We think that the observed phenomena reported by Booth and Self (1973) could be interpreted as the secondary flows triggered by a pronounced viscous heating. The rotational flows, which allow the mixing of the fluid along sections orthogonal to the direction of the flow, invoked in the model of lava flows with two thermal components by Crisp and Baloga (1990), could have a similar interpretation.

Moreover, exploration and investigations on terrestrial planets and satellites demonstrated that their lava channels are typically larger than the corresponding channels on the Earth. To account for the large sizes of lunar and martian channels Hulme $(1973,1982)$ and Carr (1974) proposed that lavas eroded the ground over which they flowed (see also Baloga et al., 1995). Our model could be used to investigate whether the action of secondary flows caused by viscous heating could have eroded the preflow surface more efficiently than laminar flows.

We wish to remark that the proposed model is applicable to slab flows, but it easily generalizable to flows in circular pipes. Moreover, modeling of magma and lava flows needs also the study of open channels with free surface and different types of thermal boundary conditions, not considered in the present study. In particular, a more realistic description should account for the temperature variations of the ambient medium, that needs the introduction of additional control parameters such as the Nusselt number which measures how much the heat transferred through the boundaries is conducted away.

Finally, an accurate study of the flow instabilities and the triggering of secondary flows needs the solution of the complete transport equations in 2-D or 3-D, which is the subject of a further work in preparation.

\section{Conclusions}

The effects of viscous heat generation in fluids with strongly temperature-dependent viscosity such as silicate melts are investigated. These effects can play an important role in the dynamics of magma flow in conduits and lava flows in tubes. In fact, viscous friction generates a local increase in temperature near the tube walls, with consequent increase of the fluid velocity because of the viscosity decrease. In the case of infinitely long tubes, one-dimensional models predict that viscous heating can lead to a positive feed-back known as "thermal runaway". Actually, as described by 2-D and 3D models, this feed-back causes the activation of other degrees of freedom, with the production of local instabilities and the triggering of secondary flows. However, the typical high value of the Peclét number in magma flows does not allow the assumption of an infinitely long tube (from the thermal point of view), and a model for magma flow in tubes of finite length is needed, as described in the paper. In general, this process is controlled by the value of the Nahme number, the flow rate, the Peclét number, and the thermal conditions at the inlet. By adopting such a model, and typical values of the parameters for lava flows in tubes, we find that starting from a constant temperature and parabolic velocity profile at the tube inlet, viscous heating causes the increase of the temperature near the walls, with a consequent local viscosity decrease. This can lead to the formation of a plug-like velocity profile, commonly observed in lava flows, and explained only by assuming a Binghamian rheology. Moreover, the presence of an inflex in the velocity profile near the walls can lead to the formation of local flow instabilities, even at low Reynolds numbers. The assumption of isothermal boundary conditions at the walls, although simplifying, is probably too restrictive; however, by assuming adiabatic conditions, the qualitative character of the flow does not seem to change dramatically in tubes of finite length. We hope that this work will provide a motivation for further theoretical and field investigations of viscous heating effects in magma flows. In particular, the formation of vortices in active lava flows at low Reynolds numbers, thermal erosion, temperature and velocity profile. Moreover, the effects viscous dissipation in volcanic conduits could have an important role on the dynamics of the both effusive and explosive eruptions.

Acknowledgements. This work was supported by the European Commission (Contract ENV4-CT98-0713), and by the Gruppo Nazionale per la Vulcanologia (INGV). We are grateful to all the researchers who contributed to improve the manuscript with their helpful suggestions, and in particular to A. Neri. Finally we wish to thank S. Baloga and the anonymous referees of this paper.

\section{References}

Anderson, O. and Perkins, P.: Runaway temperatures in the asthenosphere resulting from viscous heating, J. Geophys. Res., 79, 2136-2138, 1974.

Baloga, S. and Pieri, D.: Time-dependent lava flows, J. Geophys. Res., 91, 9543-9552, 1986.

Baloga, S., Spudis, P., and Guest, J.: The dynamics of rapidly emplaced terrestrial lava flows and implications for planetary volcanism, J. Geophys. Res., 100, 24 509-24 519, 1995.

Becker, L. and McKinley, G.: The stability of viscoelastic creeping plane shear flows with viscous heating, J. Non-Newtonian Fluid Mech., 92, 109-133, 2000.

Booth, B. and Self, S.: Rheological features of the 1971 Mount Etna lavas, Phil. Trans. R. Soc. Lond., A., 274, 99-106, 1973.

Bruce, P. and Huppert, H.: Thermal control of basaltic fissure eruptions, Nature, 342, 665-667, 1989.

Calvari, S. and Pinkerton, H.: Formation of lava tubes and extensive flow field during the 1991-1993 eruption of Mount Etna, J. Geophys. Res., 103, 27 291-27 301, 1998.

Calvari, S. and Pinkerton, H.: Lava tube morphology on Etna and evidence for lava flow emplacement mechanisms, J. Volcanol. Geotherm. Res., 90, 263-280, 1999. 
Carr, M.: The role of lava erosion in the formation of lunar rilles and martian channels, Icarus, 22, 1-23, 1974.

Costa, A. and Macedonio, G.: Nonlinear phenomena in fluids with temperature-dependent viscosity: An hysteresis model for magma flow in conduits, Geophys. Res. Lett., 29(10), 14021405, 2002.

Craik, A.: The stability of plane Couette flow with viscosity stratification, J. Fluid Mech., 36, 687-693, 1969.

Crisp, J. and Baloga, S.: A model for lava flows with two thermal components, J. Geophys. Res., 95, 1255-1270, 1990.

Crisp, J. and Baloga, S.: Influence of crystallization and entrainment of cooler material on the emplacement of basaltic aa lava flows, J. Geophys. Res., 99, 11 819-11 831, 1994.

Danes, Z.: Dynamics of lava flows, J. Geophys. Res., 77, 41430 1432, 1972.

Dragoni, M.: A dynamical model of lava flows cooling by radiation, Bull. Volcanol., 51, 88-95, 1989.

Dragoni, M., Piombo, A., and Tallarico, A.: A model for the formation of lava tubes by roofing over a channel, J. Geophys. Res., 100, 8435-8447, 1995.

Eckert, E. and Faghri, M.: Viscous heating of high Prandtl number fluids with temperature-dependent viscosity, Int. J. Heat Mass Transfer, 29, 1177-1183, 1986.

Fujii, N. and Uyeda, S.: Thermal instabilities during flow of magma in volcanic conduits, J. Geophys. Res., 79, 3367-3369, 1974.

Fujita, H.: On the nonlinear equation $\Delta u+e^{u}=0$ and $\partial v / \partial t=$ $\Delta v+e^{v}$, Bull. Am. Math. Soc., 75, 132-135, 1969.

Galili, N., Takserman-Krozer, R., and Rigbi, Z.: Heat and pressure effect in viscous flow through a pipe. 1. General formulation and basic solution, Rheol. Acta, 14, 550-557, 1975.

Greeley, R.: The role of lava tubes in Hawaiian volcanoes, in: Volcanism in Hawaii, edited by Decker, R., Wright, T., and Stauffer, P., chap. 59, pp. 1589-1602, U.S. Geol. Surv. Prof. Pap. 1350, 1987.

Greeley, R., Fagents, S., Harris, R., Kadel, S., Williams, D., and Guest, J.: Erosion by flowing lava: field evidence, J. Geophys. Res., 103, 27 325-27 346, 1998.

Gruntfest, I., Young, J., and Johnson, N.: Temperature generated by the flow of liquids in pipes, J. Appl. Phys., 35, 18-22, 1964.

Hansen, U. and Yuen, D.: Potential role played by viscous heating in thermal-chemical convection in the outer core, Geochim. Cosmochim. Acta, 60, 1113-1123, 1996.

Hulme, G.: Turbulent lava flows and the formation of lunar sinuous rilles, Mod. Geol., 4, 107-117, 1973.

Hulme, G.: A review of lava flow processes related to the formation of lunar sinuous rilles, Geophys. Surv., 5, 245-279, 1982.

John, L. and Narayanan, R.: Frictional heating in plane Couette flow, Proc. R. Soc. Lond., A, 453, 1653-1670, 1997.

Joseph, D.: Variable viscosity effects on the flow and stability of flow in channels and pipes, Phys. Fluids, 7, 1761-1771, 1964.

Kauahikaua, J., Cashman, K., Mattox, T., Heliker, C., Hon, K., Mangan, K., and Thornber, C.: Observation on basaltic lava streams in tubes from Kilauea Volcano, island of Hawaii, J. Geophys. Res., 103, 27 303-27 324, 1998.

Keszthely, L. and Self, S.: Some physical requirements for the emplacement of long basaltic lava flows, J. Geophys. Res., 103, 27 447-27 464, 1998.

Keszthelyi, L.: Measurements of the cooling at the base of pahoehoe flows, Geophys. Res. Lett., 22, 2195-2198, 1995.

Larsen, T., Yuen, D., and Malevsky, A.: Dynamical consequences on fast subducting slabs from a self-regulating mechanism due to viscous heating in variable viscosity convection, Geophys. Res.
Lett., 22, 1277-1280, 1995.

Larsen, T., Yuen, D., Smedsmo, J., and Malevsky, A.: Generation of fast timescale phenomena in thermo-mechanical processes, Phys. Earth Planet. Inter., 102, 312-322, 1997.

Lawal, A. and Kalyon, D.: Viscous heating in nonisothermal die flows of viscoplastic fluids with wall slip, Chem. Eng. Sci., 52, 1323-1337, 1997.

Lipman, P. W. and Banks, N. G.: Aa flow dynamics, Mauna Loa 1984, in: Volcanism in Hawaii, edited by Decker, R., Wright, T., and Stauffer, P., chap. 59, pp. 1527-1567, U.S. Geol. Surv. Prof. Pap. 1350, 1987.

Moore, W., Schubert, G., and Tackley, P.: Three-dimensional simulations of plume-lithosphere interaction at Hawaiian swell, Science, 279, 1008, 1998.

Ockendon, H.: Channel flow with temperature-dependent viscosity and internal viscous dissipation, J. Fluid Mech., 93, 737-746, 1979.

Park, S. and Iversen, J.: Dynamics of lava flow: thickness growth characteristics of steady two dimensional flow, Geophys. Res. Lett., 11, 641-644, 1984.

Pearson, J.: Instability in non-newtonian flow, Ann. Rev. Fluid Mech., 172, 163-181, 1976.

Pearson, J.: Variable-viscosity flows in channels with high heat generation, J. Fluid Mech., 83, 191-206, 1977.

Peterson, D., Holcomb, R., Tilling, R., and Christiansen, R.: Development of lava tubes in the light of observations at Mauna Ulu, Kilauea Volcano, Hawaii, Bull. Volcanol., 56, 343-360, 1994.

Polacci, M., Papale, P., and Rosi, M.: Textural heterogeneities in pumices from the climactic eruption of Mount Pinatubo, 15 June 1991, and implication for magma ascent dynamics, Bull. Volcanol., 63, 83-97, 2001.

Renardy, Y.: Viscosity and density stratification in vertical Poiseuille flow, Phys. Fluids, 30, 1638-1648, 1987.

Renardy, Y. and Joseph, D.: Couette flow of two fluids between concentric cylinders, J. Fluid Mech., 150, 381-394, 1985.

Schneider, J.: Einige Ergebnisse der theoretischen Untersuchung der Strömung hochviskoser Medien mit temperatur- und druckabhängigen Stoffeigenschaften in kreiszilindrischen Rohren, ZAMM, 56, 496-502, 1976.

Shaw, H.: Rheology of basalt in the melting range, J. Petrology, 10, 510-535, 1969.

Sukanek, P. and Laurence, R.: An experimental investigation of viscous heating in some simple shear flow, AIChE Journal, 20, 474 484, 1974.

Sukanek, P., Goldstein, C., and Laurence, R.: The stability of plane Couette flow with viscous heating, J. Fluid Mech., 57, 651-670, 1973.

Vasilyev, O., Ten, A., and Yuen, D.: Temperature-dependent viscous gravity currents with shear heating, Phys. Fluids, 13, 3664 3674, 2001.

White, J. and Muller, S.: Viscous heating and the stability of newtonian and viscoelastic Taylor-Couette flows, Phys. Rev. Lett., 84, 5130-5133, 2000

Yih, C.: Instability due to viscosity stratification, J. Fluid Mech., 27, 337-352, 1967.

Yueh, C. and Weng, C.: Linear stability analysis of plane Couette flow with viscous heating, Phys. Fluids, 8, 1802-1813, 1996.

Yuen, D. and Schubert, G.: Asthenospheric shear flow: thermally stable or unstable?, Geophys. Res. Lett., 4, 503-506, 1977.

Zhao, W. and Yuen, D.: The effects of adiabatic and viscous heating on plumes, Geophys. Res. Lett., 14, 1223-1227, 1987. 